\title{
Topological Properties of Incomplete WK-Recursive Networks
}

\author{
Ming-Yang Su and Gen-Huey Chen
}

Department of Computer Science and Information Engineering, National Taiwan University, Taipei, TAIWAN
Dyi-Rong Duh

Department of Electronic Engineering, Hwa Shia Junior College, Taipei, TAIWAN

\begin{abstract}
The WK-recursive networks, which were originally proposed by Vecchia and Sanges, have suffered from the rigorous restriction on the number of nodes. Like the other incomplete networks, the incomplete WK-recursive networks is proposed to relieve this restriction. In this paper, it is first shown that the structures of the incomplete WK-recursive networks are conveniently represented with multistage graphs. This representation can provide a uniform look at the incomplete WK-recursive networks. By its aid, we (1) compute the connectivities of the incomplete WK-recursive networks, (2) show that they are hamiltonian if their connectivities are greater than one, and (3) propose a sufficient and necessary condition for a hamiltonian path in an incomplete WK-recursive network with connectivity 1.
\end{abstract}

\section{Introduction}

In the recent decade, a number of networks have been proposed in the literature $[1,5,15,17,18,19]$. For these networks, many nice topological properties have been derived and many efficient algorithms have been developed. However, a major defect of these networks is that they are not truly expansible. A network is expansible if no changes with respect to node configuration and link connections are necessary when it is expanded.

We have emphasized two topological advantages, i.e., expansibility and equal degree, with the consideration of easy implementation and low cost. Recently, the WK-recursive networks [22] owning these two properties have been proposed. They offer high degree of regularity, scalability and symmetry which very well conform to a modular design and implementation of distributed systems involving a large number of computing elements. A VLSI implementation of a 16-node WK-recursive network had been realized at the Hybrid Computing Research Center [22]. Later this prototype network had been further extended to 64 nodes [23]. Some variants of the WK-recursive networks have been proposed recently $[7,8]$.

Although the WK-recursive networks own many nice properties (see $[4,6,9-11,22,23]$ ), there is a rigorous restriction on the number of their nodes. As we will see in the next section, the number of nodes contained in a WKrecursive network is restricted to $d^{t}$ where $d$ is the degree and $t$ is the level. Thus, as $d=4$, extra $3 \cdot 4^{7}=49152$ nodes are required to expand from a 7-level WK-recursive network to a 8-level WK-recursive network. Almost all of the networks mentioned earlier in this section suffered from the same problem. Therefore, some incomplete structures $[12,13,14$, 16] have been proposed as a solution to this problem.

In this paper, we define the incomplete WK-recursive networks that require the number of nodes to be a multiple of $d$, where $d$ is the size of the basic building block. Since each basic building block of the WK-recursive networks contains $d$ nodes, the incomplete WK-recursive networks can be expanded or contracted in arbitrary units of basic building blocks. We then compute the connectivities and hamiltonicity of the incomplete WK-recursive networks.

In the next section, the WK-recursive networks are reviewed and the incomplete WK-recursive networks are formally defined. The connectivities and hamiltonicity are discussed, respectivity, in Sections 3 and 4. Finally, this paper is concluded with some remarks in Section 5.

\section{WK-Recursive Networks and Incomplete WK-Recursive Networks}

The WK-recursive networks can be constructed recursively by grouping basic building blocks. Any complete graph can serve as a basic building block. For convenience, we use $\mathbf{K}(d, t)$ to denote a WK-recursive network of level $t$ whose basic building blocks are each a $d$-node complete graph, where $d>1$ and $t \geq 1 . K(d, 1)$, which is the basic building block, is a $d$-node complete graph, and $\mathrm{K}(d, t)$ for $t \geq 2$ is composed of $d \mathbf{K}(d, t-1)$ 's which are connected as a complete graph. Each node of $\mathrm{K}(d, t)$ has degree $d$ and can be uniquely identified by a sequence of $t$ digits. We define $K(d$, $t$ ) formally as follows.

Definition 2.1. The node set of $K(d, t)$ is denoted by $\left\{a_{t-1} a_{t-2} \ldots a_{1} a_{0} \mid a_{i} \in\{0,1, \ldots, d-1\}\right.$ for $\left.0 \leq i \leq t-1\right\}$. Node adjacency is defined as follows: $a_{t-1} a_{t-2 \ldots} a_{1} a_{0}$ is adjacent to (1) $a_{t-1} a_{t-2} \ldots a_{1} b$, where $0 \leq b \leq d-1$ and $b \neq a_{0}$, and (2) $a_{t-1} a_{t-2}$

$0-7803-3529-5 / 96 / \$ 5.00^{\bullet} 1996$ IEEE 
$\ldots a_{i+1} a_{i-1}\left(a_{i}\right)^{i}$ if $a_{i} \neq a_{i-1}$ and $a_{i-1}=a_{i-2}=\ldots=a_{1}=a_{0}$, where $\left(a_{i}\right)^{i}$ represents $i$ consecutive $a_{i}$ 's. The links of (1) are named substituting links and assigned label 0 . The links of (2) are named flipping links and assigned label $i$. The flipping links with label $i$ are referred to as $i$-flipping links. Besides, there are open links whose one end node is $a^{t}$, where $0 \leq a \leq d-1$, and the other end node is unspecified. The open links are labeled

Since each node is incident with $d-1$ substituting links and one flipping link (or open link), $\mathrm{K}(d, t)$ has degree $\mathrm{d}$. The structures of $K(4,1)$ and $K(4,3)$ are illustrated in Figure 1. Intuitively, the substituting links are those within basic building blocks, the $i$-flipping links each connect two embedded $\mathrm{K}(d, i)$ 's, and the open links are left for future expansion. For example, let us consider the incident links of node 311 in Figure 1. The one to node 133 is a 2-flipping link, and the others are substituting links.

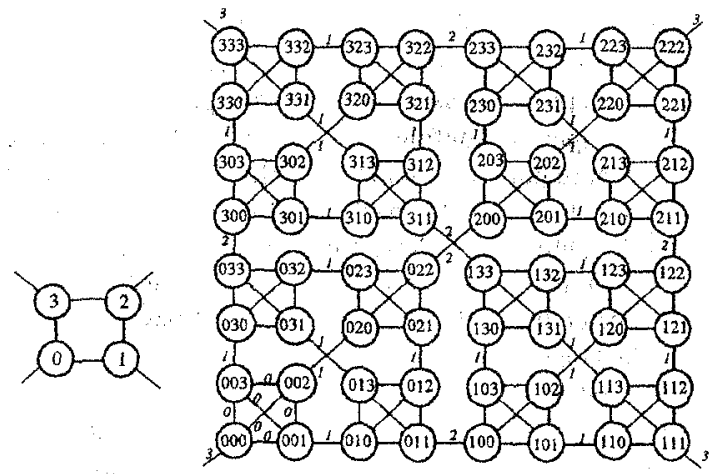

Figure 1. The structures of $K(4,1)$ and $K(4,3)$.

Definition 2.2. Define $c_{t-1} c_{t-2} \ldots c_{r} \cdot \mathrm{K}(d, r)$ to be the induced subgraph of $\mathrm{K}(d, t)$ by $\left(c_{t-1} c_{t-2} \ldots c_{r} a_{r-1} \ldots a_{1} a_{0} \mid a_{i} \in\right.$ $\{0,1, \ldots, d-1\}$ for $0 \leq i \leq r-1\}$, where $1 \leq r \leq t-1$ and $c_{t-1}, c_{t-2}$, $\ldots, c_{r}$ are all integers from $\{0,1, \ldots, d-1\}$.

In Figure 1, for example, $20 \cdot \mathrm{K}(4,1)$ is the subgraph induced by $\{200,201,202,203\}$.

Definition 2.3. Node $a_{t-1} a_{t-2} \ldots a_{1} a_{0}$ is a $k$-frontier, where $1 \leq k \leq t$, if $a_{k-1}=\ldots=a_{1}=a_{0}$.

Note that by Definition 2.3 a $k$-frontier is automatically an $l$-frontier, where $1 \leq k<k$. Both end nodes of a $k$-flipping link are $k$-frontiers. An embedded $\mathrm{K}(d, r)$ contains one $(r+1)$ frontier and $d-l, r$-frontiers.

Now, we begin to introduce the incomplete WK-tecursive networks. The incomplete WK-recursive networks are subgraphs of the WK-recursive networks. For convenience, we use $\operatorname{IK}(d, t)$ to denote an incomplete WK-recursive network with $N$ nodes, where $d^{t-1}<N<d^{t}$ is a multiple of $d$. The restriction to $N$ is because $K(d, 1)$ remains the basic building block for $\operatorname{IK}(d, t)$. The structure of $\operatorname{IK}(d, t)$ with $N$ nodes can be uniquely determined by the associated coefficient vector, as defined betow.

Definition 2.4. The coefficient vector associated with an $N$-node $\mathrm{IK}(d, t)$ is a $(t-1)$-tuple $\left(b_{t-1}, b_{t-2}, \ldots, b_{1}\right)$ satisfying $N=b_{t-1} d^{t-1}+b_{t-2} d^{t-2}+\ldots+b_{1} d$, where $1 \leq b_{t-1} \leq d-1$ and $0 \leq b_{i} \leq d-1$ for $1 \leq i \leq t-2$.

Let $V\left(b_{t-1}, b_{t-2}, \ldots, b_{1}\right)$ denote the node set of $\operatorname{IK}(d, t)$ with coefficient vector $\left(b_{t-1}, b_{t-2}, \ldots, b_{1}\right)$ and $V(i \cdot \mathrm{K}(d, t-1))$ denote the node set of $i \cdot \mathrm{K}(d, t-1)$, where $0 \leq i \leq d-1$. The set $V\left(b_{t-1}, b_{t-2}, \ldots, b_{1}\right)$ can be defined recursively as follows

$V\left(b_{t-1}, b_{t-2}, \ldots, b_{1}\right)=V(0 \cdot \mathrm{K}(d, t-1))+V(1 \cdot \mathrm{K}(d, t-1))+\ldots$ $+V\left(\left(b_{t-1}-1\right) \cdot \mathrm{K}(d, t-1)\right)+V\left(b_{t-1} \cdot\left(b_{t-2}, b_{t-3}, \ldots, b_{1}\right)\right)$,

where +denotes a union operation and $b_{t-1} \cdot\left(b_{t-2}, b_{t-3}, \ldots, b_{1}\right)$ represents an IK $(d, t-1)$ with coefficient vector $\left\langle b_{t-2}, b_{t-3}, \ldots\right.$ $\left.b_{1}\right)$ that is contained in $b_{t-1} \cdot K(d, t-1)$ provided $b_{t-2} \neq 0$. If $b_{t-2}=$ $b_{t-3}=\ldots=b_{r}=0$ and $b_{r-1} \neq 0$, where $1<r \leq t-2$, then $b_{t-1} \cdot\left(b_{t-2}\right.$, $\left.b_{t-3}, \ldots, b_{1}\right)$ represents an $\operatorname{IK}(d, r)$ with coefficient vector ( $\left.b_{r-1}, b_{r-2}, \ldots, b_{1}\right)$ that is contained in $b_{t-1} 0^{t-r-1} \cdot \mathrm{K}(d, r)$.

For example, the coefficient vector of $\operatorname{IK}(5,6)$ with 8225 nodes is $(2,3,0,4,0)$ and its node set can be expressed as follows.

$V(2,3,0,4,0)$

$=V(0 \cdot \mathrm{K}(5,5))+V(1 \cdot \mathrm{K}(5,5))+V(2 \cdot(3,0,4,0))$

$=V(0 \cdot \mathrm{K}(5,5))+V(1 \cdot \mathrm{K}(5,5))+V(20 \cdot \mathrm{K}(5,4))+V(21 \cdot \mathrm{K}(5$, 4) $)+V(22 \cdot K(5,4))+V(23 \cdot(0,4,0))$

$=V(0 \cdot \mathrm{K}(5,5))+V(1 \cdot \mathrm{K}(5,5))+V(20 \cdot \mathrm{K}(5,4))+V(21 \cdot \mathrm{K}(5$, $4))+V(22 \cdot K(5,4))+V(230 \cdot(4,0))$

$=V(0 \cdot \mathrm{K}(5,5))+V(1 \cdot \mathrm{K}(5,5))+V(20 \cdot \mathrm{K}(5,4))+V(21 \cdot \mathrm{K}(5$ 4) $)+V(22 \cdot \mathrm{K}(5,4))+V(2300 \cdot \mathrm{K}(5,2))+V(2301 \cdot \mathrm{K}(5$, $2))+V(2302 \cdot K(5,2))+V(2303 \cdot K(5,2))$

The structure of $\operatorname{IK}(d, t)$ with coefficient vector $\left(b_{t-1}, b_{t-2}\right.$, $\left.\ldots, b_{1}\right)$ is defined as follows

Definition 2.5. $\mathrm{IK}(d, t)$ with coefficient vector $\left(b_{t-1}\right.$, $\left.b_{t-2}, \ldots, b_{1}\right)$ is the induced subgraph of $\mathrm{K}(d, t)$ by $V\left(b_{t-1}\right.$ $\left.b_{t-2}, \ldots, b_{1}\right)$.

See Figure 2 where the structure of $\operatorname{IK}(4,3)$ with coefficient vector $(3,2)$ is shown.

\section{Connectivity}

The connectivity of a connected network is defined as the minimum number of nodes whose removal can result in the network disconnected. Connectivity is usually adopted as a measure for fault tolerance in networks because Menger's theorem [3] states that the number of node-disjoint paths between two nodes of a network is at least its connectivity. Since $\operatorname{IK}(d, t)$ is a subgraph of $\mathrm{K}(d, t)$, the connectivity of the 
former is not greater than the connectivity of the latter. The connectivity of $K(d, t)$ is known to he $d-1$ [4]. In this section. the connectivity of $[\mathrm{K}(d, t)$ is computed. Firsl, some necessary detinitions and lemmas are introduced.

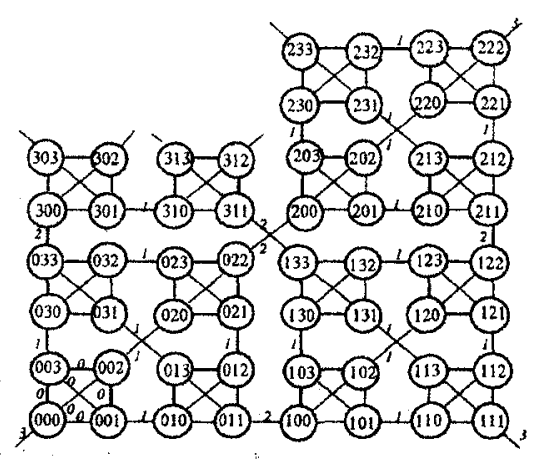

Figure 2. The structures of $\operatorname{IK}(4,3)$ with coefficient vector $(3,2)$.

According to Definition 2.5 , IK $(d, t)$ with coefficient vector $\left(b_{t-1}, b_{t-2}, \ldots, b_{1}\right)$ contains $b_{t-1}$ embedded $\mathrm{K}(d, t-1)$ 's, $b_{t-2}$ embedded $K(d, t-2)$ 's, $\ldots$, and $b_{1}$ embedded $K(d, 1)$ 's. For $1 \leq i \leq t-1$, the $b_{i}$ embedded $K(d, i)$ 's are $b_{t-1} b_{t-2} \ldots$ $b_{i+1} 0 \cdot \mathrm{K}(d, i), b_{t-1} b_{t-2}, \ldots b_{i+1} l \cdot \mathrm{K}(d, i), \ldots$, and $b_{t-1} b_{t-2} \ldots$ $b_{i+1}\left(b_{i}-1\right) \cdot K(d, i)$. Let $G_{i}$ represent the induced subgraph of IK $(d, t)$ with coefficient vector $\left(b_{t-1}, b_{t-2}, \ldots, b_{1}\right)$ by $V\left(b_{t-1}\right.$ $\left.b_{t-2} \ldots b_{i+1} 0 \cdot \mathbf{K}(d, i)\right)+V\left(b_{t-1} b_{t-2} \ldots b_{i+1} 1 \cdot \mathbf{K}(d, i)\right)+\ldots+V\left(b_{t-1}\right.$ $\left.b_{t-2 \ldots} . b_{i+1}\left(b_{i}-1\right) \cdot \mathrm{K}(d, i)\right)$, and $R_{n}^{m}$, where $1 \leq n \leq m \leq t-1$, the connectivity of $G_{m}+G_{m-1}+\ldots+G_{n}$. Then, $R_{1}^{i-1}$ is the connectivity of $\mathrm{IK}(d, t)$ with coefficient vector $\left(b_{t-1}, b_{t-2}, \ldots\right.$, $b_{1}$ ). In Figure 2, for example, we have $R_{2}^{2}=2, R_{1}^{1}=1$, and the connectivity of the IK $(4,3)$ is $R_{1}^{2}=2$.

For easy reference, we refer to $b_{t-1} b_{t-2 \ldots} b_{i+1} r \cdot \mathrm{K}(d, i)$ as the $(r+1)$ th $K(d, i)$ within $G_{i}$ in the subsequent discussion, where $0 \leq r \leq b_{i}-1$. Besides, a coefficient vector $\left(b_{t-1}, b_{t-2}, \ldots\right.$, $\left.b_{1}\right)$ is written as $\left(b_{t-1}, b_{t-2}, \ldots, b_{i}, *\right)$, provided $b_{1}=b_{2}=\ldots$ $=b_{i-1}=0$ and $b_{i} \neq 0$. For example, $(2,3,0,4,0)$ is written as $(2,3,0,4, *)$, and $(2,3,4)$ is written as $(2,3,4, *)$.

Lemma 3.1. For $\operatorname{IK}(d, t)$ with coefficient vector $\left(b_{t-1}\right.$, $\left.b_{t-2}, \ldots, b_{i}, *\right), R_{m}^{m}=b_{m}-1$ if $b_{m} \geq 2$, where $1 \leq i \leq m \leq t-1$.

Proof. $G_{m}$ can be regarded as a $b_{m}$-node complete graph with each node being a $\mathrm{K}(d, m)$. The connectivity of $\mathrm{K}(d, m)$ is known to be $d-1$. Since at least $b_{m}-1(<d-1)$ nodes have to be removed in order to disconnect a $b_{m}$-node complete graph, the connectivity of $G_{m}$ is $b_{m}-1$. Hence, $R_{m}^{m}=b_{m}-1$. Q.E.D.

Lemma 3.2. For $\operatorname{IK}(d, t)$ with coefficient vector $\left(b_{t-1}\right.$, $\left.b_{i-2}, \ldots, b_{i}, *\right), R_{m-1}^{m}=\min \left(b_{m-1}, b_{m}\right)$ if $b_{m-1} \geq 1$ and $b_{m} \geq 1$, where $1 \leq i<m \leq t-1$.
Proof. We first assume $b_{m}<b_{m-1}$. For $0 \leq j \leq b_{m}-1$. there is an $m$-Ilipping link between $b_{t-1} b_{t-2} \ldots b_{m+1} j \cdot \mathrm{K}(d, m)$ and $b_{t-}$ ${ }_{1} b_{t-2} \ldots b_{m j} \cdot \mathbf{K}(d, m-1)$. There are several possibilities (and their combinations) to disconnect $G_{m}+G_{m-t}$. To isolate one or more nodes from a $\mathrm{K}(d, m-1)$ or a $\mathrm{K}(d, m)$ requires removing at least $d-1$ nodes. To isolate one or more $\mathrm{K}(d, m)$ 's from $G_{m}$ requires removing at least $b_{m}$ nodes. To isolate one or more $\mathrm{K}(d, m-1)$ 's from $G_{m-1}$ requires removing at least $b_{m-1}-1$ nodes. To separate $G_{m-1}$ from $G_{m}$ requires removing at least $b_{m}$ nodes. Hence, the connectivity of $G_{m}+G_{m-1}$ is $b_{m}$.

With similar arguments, the connectivity of $G_{m}+G_{m-1}$ can be proved to be $b_{m}$ if $b_{m}=b_{m-1}$, and $b_{m-1}$ if $b_{m}>b_{m-1}$. This completes the proof.

Q.E.D.

Lemma 3.3. For $\operatorname{IK}(d, t)$ with coefficient vector $\left(b_{t-1}\right.$, $\left.b_{i-2}, \ldots, b_{i}, *\right), R_{m-1}^{m+1}=b_{m}+1$ if $b_{m+1}>b_{m}$ and $b_{m}<b_{m-1}$, and $\min \left\{b_{m+1}, b_{m}, b_{m-1}\right\}$ else, where $b_{m+1} \geq 1, b_{m} \geq 0, b_{m-1} \geq 1$, and $1 \leq i<m \leq t-2$.

Proof. There are five cases: (1) $b_{m+1} \leq b_{m} \leq b_{m-1}$; (2) $b_{m+1} \geq b_{m} \geq b_{m-1}$; (3) $b_{m+1}<b_{m}$ and $b_{m}>b_{m-1}$; (4) $b_{m+1}>b_{m}$, $b_{m}<b_{m-1}$, and $b_{m} \neq 0$; (5) $b_{m}=0$, to be considered.

Case 1. $b_{m+1} \leq b_{m} \leq b_{m-1}$.

Note that for $i<j \leq t-1$, there are min $\left\{b_{j}, b_{j-1}\right\} j$-flipping links coninecting $G_{j}$ and $G_{j-1}$. By Lemma $3.2, R_{m}^{m+1}=b_{m+1}$ and $R_{m-1}^{m}=b_{m}$. Since no link exists between $G_{m+1}$ and $G_{m-1}$ (see Figure 3(a) where the links among each $G_{j}$ are omitted), the connectivity of $G_{m+1}+G_{m}+G_{m-1}$ is $b_{m+1}$.

Case 2. $b_{m+1} \geq b_{m} \geq b_{m-1}$.

By Lemma 3.2, $R_{m}^{m+1}=b_{m}$ and $R_{m-1}^{m}=b_{m-1}$. The connectivity of $G_{m+1}+G_{m}+G_{m-1}$ is $b_{m-1}$ with the arguments similar to Case 1 .

Case 3. $b_{m+1}<b_{m}$ and $b_{m}>b_{m-1}$

By Lemma $3.2, R_{m}^{m+1}=b_{m+1}$ and $R_{m-1}^{m}=b_{m-1}$. The connectivity of $G_{m+1}+G_{m}+G_{m-1}$ is $\min \left\{b_{m+1}, b_{m-1}\right\}$ with the arguments similar to Case 1.

Case 4. $b_{m+1}>b_{m}, b_{m}<b_{m-1}$, and $b_{m} \neq 0$.

By Lemma 3.2, $R_{m}^{m+1}=b_{m}$ and $R_{m-1}^{m}=b_{m}$. There are $b_{m}$ $(m+1)$-flipping links, i.e., $\left(b_{t-1} b_{t-2} \ldots b_{m+2} 0\left(b_{m+1}\right)^{m+1}, b_{t-1}\right.$ $\left.b_{t-2} \ldots b_{m+2} b_{m+1} 0^{m+1}\right),\left(b_{t-1} b_{t-2} \ldots b_{m+2} 1\left(b_{m+1}\right)^{m+1}, b_{t-1} b_{t-2} \ldots\right.$ $\left.b_{m+2} b_{m+1} 1^{m+1}\right), \ldots$, and $\left(b_{t-1} b_{t-2} \ldots b_{m+2}\left(b_{m}-1\right)\left(b_{m+1}\right)^{m+1}, b_{t-1}\right.$ $b_{t-2 \ldots} \ldots b_{m+2} b_{m+1}\left(b_{m}-1\right)^{m+1}$ ), between $G_{m+1}$ and $G_{m}$ (sce Figure 3(b)). Besides, there is exactly one ( $m+1)$-flipping link between $G_{m+1}$ and $G_{m-1}$ as explained as follows. There is an $(m+1)$-flipping link, i.e., $\left(b_{t-1} b_{t-2} \ldots b_{m+2} b_{m}\left(b_{m+1}\right)^{m+1}, b_{t-1}\right.$ $\left.b_{t-2} \ldots b_{m+2} b_{m+1}\left(b_{m}\right)^{m+1}\right)$, connecting $b_{t-1} b_{t-2} \ldots b_{m+2} b_{m} \cdot \mathbf{K}(d$, $m+1)$ and $b_{t-1} b_{t-2} \ldots b_{m+2} b_{m+1} b_{m} b_{m} \cdot \mathbf{K}(d, m-1)$ which belong to $G_{m+1}$ and $G_{m-1}$, respectively. For $j>b_{m}$, the link $\left(b_{t-1} b_{t-2}\right.$ $\left.\ldots b_{m+2} j\left(b_{m+1}\right)^{m+1}, b_{t-1} b_{t-2} \ldots b_{m+2} b_{m+1} j^{m+1}\right)$ does not exist 
because $b_{t-1} b_{t-2} \ldots b_{m+2} b_{m+1} j^{m+1}$ is not a node in the $\operatorname{IK}(d, t)$. Hence, the connectivity of $G_{m+1}+G_{m}+G_{m-1}$ is $b_{m}+1$.

Case $5 . b_{m}=0$.

This case is a degenerated case of Case 4 (see Figure $3(c))$. The connectivity of $G_{m+1}+G_{m}+G_{m-1}$ is 1 Q.E.D.

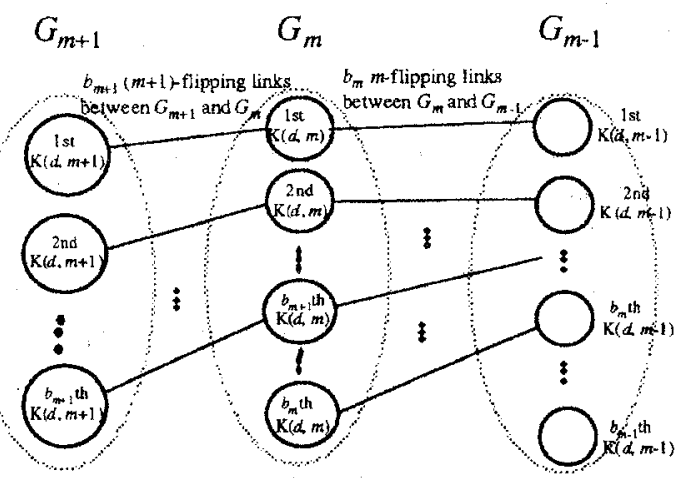

(a)

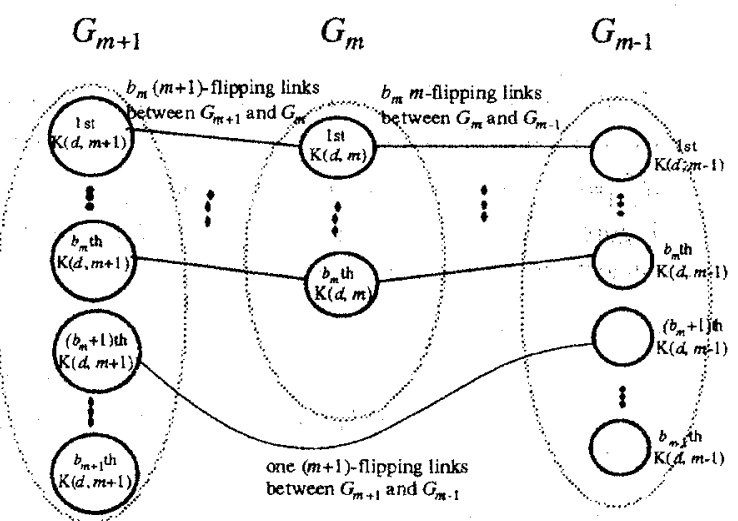

(b)

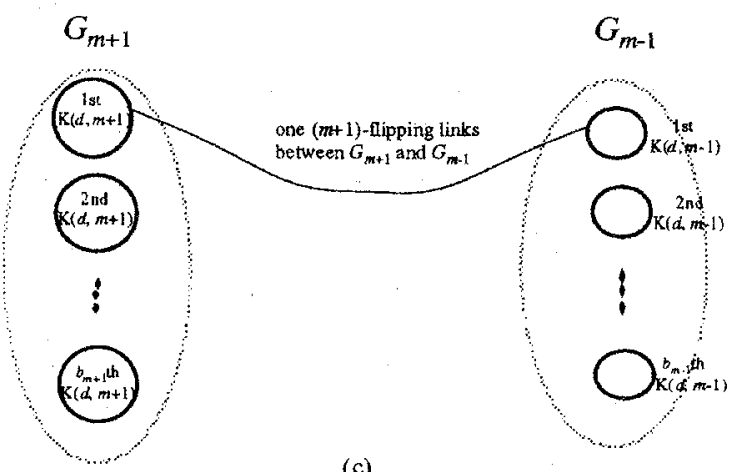

(c)

Figure 3. The proof of Lemma 3.3. (a) Case 1. (b) Case 4. (c) Case 5 .
Lemma 3.4. For $\operatorname{IK}(d, t)$ with coefficient vector $\left(b_{t-1}\right.$, $\ldots, b_{m}, r, \ldots, r, b_{n}, \ldots, b_{i}, *$ ) (i.e., $b_{m-1}=b_{m-2}=\ldots$ $\left.=b_{n+1}=r\right)$, where $1 \leq i \leq n<m \leq t-1, m \geq n+2, b_{m}>r, b_{n}>r$, and $0 \leq r \leq d-2, R_{n}^{m}=r+1$. Moreover, there exists exactly one $m$ flipping link between $G_{m}$ and $G_{n}$, and no other link exists between $G_{x}$ and $G_{\gamma}$, where $m \leq x \leq t-1$ and $i \leq y \leq n$.

Proof. By Lemma 3.2, $R_{m-1}^{m}=R_{m-2}^{m-1}=\ldots=R_{n}^{n+1}=r$. It is not difficult to see that for $n+1 \leq k \leq m-2$ and $n+2 \leq k \leq m-1$, no link exists between $G_{m}$ and $G_{k}$ and between $G_{n}$ and $G_{l}$. With the arguments similar to Case 4 in the proof of Lemma 3.3, there is exactly one $m$-flipping link, i.e., $\left(b_{t-1} b_{t-2} \ldots b_{m+1} r\left(b_{m}\right)^{m}\right.$, $\left.b_{t-1} b_{:-2} \ldots b_{m+1} b_{m} r^{m}\right)$, between $G_{m}$ and $G_{n}$. Hence, the connectivity of $G_{m}+G_{m-1}+\ldots+G_{n}$ is $r+1$.

Then we proceed to show that no other link exists between $G_{x}$ and $G_{y}$. We first assume $x \neq m$ and $y \neq n$. If a ( $x$ flipping) link exists between $G_{x}$ and $G_{y}$, its two end nodes should be $b_{t-1} b_{t-2 \ldots} b_{x+1} \alpha\left(b_{x}\right)^{x}$ and $b_{t-1} b_{t-2 \ldots} \ldots b_{x+1} b_{x}(\alpha)^{x}$ for some $\alpha>r$. However, since $x>m$ and $\alpha>r, b_{t-1} b_{t-2}$.. $b_{x+1} b_{x}(\alpha)^{x}$ is not a node in the IK $(d, t)$. Similarly, it can be proved that no link exists between $G_{x}$ and $G_{y}$ if $x=m$ and $i \leq y<n$ or $m<x \leq t-1$ and $y=n$.

Q.E.D.

Theorem 3.1. For $\operatorname{IK}(d, t)$ with coefficient vector $\left(b_{t-1}\right.$ $\left.b_{t-2}, \ldots, b_{\mathrm{i}}, *\right), 1 \leq i \leq t-1$, its connectivity, i.e., $R_{i}^{i-1}$ can be determined as follows.

(1) If $i=t-1$, then $R_{i}^{t-1}=b_{t-1}-1$

(2) Otherwise, letting $k=\min \left\{b_{t-1}, b_{t-2}, \ldots, b_{i}\right\}, R_{i}^{t-1}=k$ if $b_{t-1}=k$ or $b_{i}=k$, and $k+1$ else.

Proof. We prove this theorem by induction on $i$. Induction basis. Lemmas 3.1, 3.2, and 3.3 show the validity of the theorem for $i=t-1, t-2$, and $t-3$, respectively.

Induction hypothesis. Assume the theorem is valid for $i=m+1$, where $1 \leq m \leq t-4$. Let $k^{\prime}=\min \left\{b_{t-1}, b_{t-2}, \ldots, b_{m+1}\right\}$. Induction step. We now discuss the case of $i=m$. Three cases are considered according to the value of $k^{\prime}$.

Case 1. $k^{\prime}=b_{t-1}$

In this case $R_{m+1}^{t-1}=b_{t-1}$. Since $b_{t-1} \leq b_{t-2}$, no link emits from $G_{t-1}$ to $G_{j}$, where $m \leq j \leq t-3$. Consequently, removing $b_{t-1}$ nodes will seperate $G_{t-1}$ from $G_{t-2}+G_{t-3}+\ldots+G_{m}$. We first assume $b_{m+1} \leq b_{m}$. By Lemma 3.2 we have $R_{m}^{m+1}=b_{m+1}$. There are three possibilities (or their combinations) to disconnect $G_{t-1}+G_{t-2}+\ldots+G_{m}$. One is to disconnect $G_{m}$ which requires removing at least $b_{m+1}$ nodes. Another is to seperate $G_{m}$ from $G_{t-1}+G_{t-2}+\ldots+G_{m+1}$ which requires removing at least $b_{m+1}$ nodes. The other is to disconnect $G_{t-1}+G_{t-2}+\ldots+G_{m+1}$ which requires removing at least $b_{t-1}$ nodes. Hence, $R_{m}^{t-1}=\min \left\{b_{t-1}, b_{m+1}\right\}=b_{t-1}$. Note that $b_{t-1}=\min \left\{b_{t-1}, b_{t-2}, \ldots\right.$, $\left.b_{m+1}, b_{m}\right\}$

On the other hand, if $b_{m+1}>b_{m}, R_{m}^{m+1}=b_{m}$ by Lemma 3.2, and no link emits from $G_{m}$ to $G_{l}$, where $m+2 \leq l \leq t-1$. 
Similarly, $R_{m}^{t-1}$ can be determined as $\min \left\{b_{t-1}, b_{m}\right\}$. Note that $\operatorname{since} b_{t-1}=\min \left\{b_{t-1}, b_{t-2}, \ldots, b_{m+1}\right\}, \min \left\{b_{t-1}, b_{m}\right\}=\min \{$ $\left.b_{1-1}, b_{1-2}, \ldots, b_{m+1}, b_{m}\right\}$.

Case 2. $k^{\prime}=b_{m+1}$.

In this case $R_{m+1}^{t-1}=b_{m+1}$. If $b_{t-1}=b_{t-2}=\ldots=b_{m+1}$, the discussion is the same as Case 1 because $b_{t-1}=k^{\prime}$. Otherwise, let $j=\min \left\{l \mid m+2 \leq l \leq t-1\right.$ and $\left.b_{l}>b_{m+1}\right\}$. If $b_{m+1}<b_{m}$, by Lemma 3.4 there is a $j$-flipping link between $G_{m}$ and $G_{j}$. Also note that no link exists between $G_{m}$ and $G_{s}$ for $s \neq j$ and $s \neq m+1$. If $b_{m+1} \geq b_{m}$, no link exists between $G_{m}$ and $G_{s}$ for $s \neq m+1$. With the arguments similar to Case 1 , it can be proved that $R_{m}^{t-1}=b_{m+1}+1$ and $b_{m+1}=\min \left\{b_{t-1}, b_{t-2}, \ldots, b_{m+1}\right.$, $\left.b_{m}\right\}$ if $b_{m+1}<b_{m}$, and $R_{m}^{t-1}=b_{m}$ and $b_{m}=\min \left\{b_{t-1}, b_{t-2}, \ldots\right.$, $\left.b_{m+1}, b_{m}\right\}$ else.

Case 3. $k^{\prime} \neq b_{t-1}$ and $k^{\prime} \neq b_{m+1}$

We assume $k^{\prime}=b_{r}$, where $m+1<r<t-1$. In this case $R_{m+1}^{t-1}=b_{r}+1$. Let $j=\max \left\{l \mid m<l<r\right.$ and $\left.b_{l}>b_{r}\right\}$. By Lemma 3.4, no link exists between $G_{m}$ and $G_{s,}$ where $j<s \leq t-1$. With the arguments similar to Case 1 , it can be proved that (1) if $b_{m+1} \leq b_{m}, R_{m}^{t-1}=b_{r}+1$ and $b_{r}=\min \left\{b_{t-1}, b_{t-2}, \ldots, b_{r}, \ldots, b_{m+1}\right.$, $\left.b_{m}\right\} ;$; (2) if $b_{m+1}>b_{m}$ and $b_{m}<b_{r}+1, R_{m}^{i-1}=b_{m}$ and $b_{m}=\min \left\{b_{t-1}\right.$, $\left.b_{t-2}, \ldots, b_{r}, \ldots, b_{m+1}, b_{m}\right\} ;$ (3) if $b_{m+1}>b_{m}$ and $b_{m} \geq b_{r}+1$. $R_{m}^{t-1}=b_{r}+1$ and $b_{r}=\min \left\{b_{t-1}, b_{t-2}, \ldots, b_{r}, \ldots, b_{m+1}, b_{m}\right\}$.

Q.E.D.

We have the following corollary immediately.

Corollary 3.1. For $\operatorname{IK}(d, t)$ with coefficient vector ( $\left.b_{t-1}, b_{t-2}, \ldots, b_{i}, *\right)$, letting $k=\min \left\{b_{m}, b_{m-1}, \ldots, b_{n}\right\}$, where $1 \leq i \leq n<m \leq t-1, b_{m} \neq 0$, and $b_{n} \neq 0, R_{n}^{m}=k$ if $b_{m}=k$ or $b_{n}=k$, and $k+1$ else.

For $i \leq n<m \leq t-1$, an $m$-flipping link between $G_{m}$ and $G_{n}$ is called a jumping $m$-flipping link if $m-n>1$. Note that by Lemma 3.4 the flipping links of an $\operatorname{IK}(d, t)$ with coefficient vector $\left(b_{t-1}, b_{t-2}, \ldots, b_{i}, *\right)$ can be determined from its coefficient vector. We take $\operatorname{IK}(6,10)$ with coefficient vector $(4,3,4,2,1,1,3, *)$ as an illustrative example. There are two jumping flipping links. One is between the 4th $K(6,9)$ within $G_{9}$ and the 4 th $K(6,7)$ within $G_{7}$, and the other is between the 2 nd $\mathrm{K}(6,6)$ within $G_{6}$ and the 2nd $\mathrm{K}(6,3)$ within $G_{3}$. An easy way to determine jumping flipping links is that for any local minimal value, say $b_{r}$, in the sequence $b_{t-1}, b_{t-2}, \ldots, b_{i}$, there exists a jumping ( $m$-flipping) link between $G_{m}$ and $G_{n}$, where $i<r<t-1$ and $m=\min \{l \mid r<l \leq t-1$ and $\left.b_{l}>b_{r}\right\}$ and $n=\max \left\{l \mid i \leq l<r\right.$ and $\left.b_{l}>b_{r}\right\}$, if $m$ and $n$ exist. This link connects the $\left(b_{r}+1\right)$ th $\mathrm{K}(d, m)$ and the $\left(b_{r}+1\right)$ th $\mathrm{K}(d$, $n)$. All non-jumping flipping links exist between $G_{m}$ and $G_{m-1}$, where $i<m \leq t-1$. More specifically, $\min \left\{b_{m}, b_{m-1}\right\} m$ flipping links connect the $j$ th $\mathrm{K}(d, m)$ within $G_{m}$ and the $j$ th $\mathrm{K}(d, m-1)$ within $G_{m-1}$ for all $1 \leq j \leq \min \left\{b_{m}, b_{m-1}\right\}$.

\section{Hamiltonicity}

A cycle (path) in a network is called a hamiltonian cycle (path) if it contains every node of the network exactly once. A network is hamiltonian if it contains a hamiltonian cycle. A hamiltonian network can embed a ring with unit expansion and unit dilation. In this section, we show that $\operatorname{IK}(d, t)$ with connectivity greater than one is hamiltonian. Moreover, we propose a sufficient and necessary condition for a hamiltonian path in an $\operatorname{IK}(d, t)$ with connectivity one. Chen and Duh [4] have shown that $\mathbf{K}(2, t)$ contains a hamiltonian path, and $\mathbf{K}(d$, t) contains a hamiltonian cycle for $d \geq 3$. Moreover, they have shown the following result.

Lemma 4.1. [4] There is one hamiltonian path between any two $t$-frontiers in $\mathrm{K}(d, t)$.

Since $\operatorname{IK}(2, t)$ has a linear structure, it contains a hamiltonian path. In this section, we concentrate our attention on the hamiltonicity of $\operatorname{IK}(d, t)$ for $d \geq 3$. First we adapt Lemma 4.1 to $\operatorname{IK}(d, t)$.

Lemma 4.2. There are two hamiltonian paths, one between $0^{t}$ and $1^{t}$ and the other between $0\left(b_{t-1}\right)^{s-1}$ and $1\left(b_{t-1}\right)^{t-1}$, in $\mathrm{IK}(d, t)$ with coefficient vector $\left(b_{t-1}, *\right)$, where $b_{t-1} \geq 2$.

Proof. A hamiltonian path between $0^{t}$ and $1^{t}$ can be constructed as follows:

$$
\begin{aligned}
& 0^{t} \rightarrow(\mathrm{H}, t-1) \quad 0\left(b_{t-1}-1\right)^{t-1} \rightarrow\left(b_{t-1}-1\right) 0^{t-1} \rightarrow(\mathrm{H}, t-1) \\
& \left(b_{t-1}-1\right)\left(b_{t-1}-2\right)^{t-1} \rightarrow\left(b_{t-1}-2\right)\left(b_{t-1}-1\right)^{t-1} \rightarrow \rightarrow_{(\mathrm{H}, t-1)}\left(b_{t-1}-2\right) \\
& \left(b_{t-1}-3\right)^{t-1} \rightarrow\left(b_{t-1}-3\right)\left(b_{t-1}-2\right)^{t-1} \rightarrow_{(\mathrm{H}, t-1)} \cdots \rightarrow_{(\mathrm{H}, t-1)} \\
& 21^{t-1} \rightarrow 12^{t-1} \rightarrow(\mathrm{H}, t-1) 1^{t}
\end{aligned}
$$

where $\rightarrow$ indicates a flipping link and $\rightarrow_{(\mathrm{H}, t-1)}$ indicates a hamiltonian path in a $\mathrm{K}(d, t-1)$. A hamiltonian path between $O\left(b_{t-1}\right)^{t-1}$ and $1\left(b_{t-1}\right)^{t-1}$ can be obtained by substituting $O($ $\left.b_{t-1}\right)^{t-1}$ and $1\left(b_{t-1}\right)^{t-1}$, respectively, for $0^{t}$ and $1^{t}$ in the construction above. The correctness is assured by Lemma 4.1 .

Q.E.D.

Lemma 4.3. There are two node-disjoint paths, one between $0\left(b_{t-1}\right)^{t-1}$ and $0^{t}$ and the other between $1\left(b_{t-1}\right)^{t-1}$ and $1^{t}$, in $\operatorname{IK}(d, t)$ with coefficient vector $\left(b_{t-1}, *\right)$, where $b_{t-1} \geq 2$, such that they contain every node of the IK $(d, t)$ exactly once.

Proof. There are $b_{t-1} \mathrm{~K}(d, t-1)$ 's, i.e., $0 \cdot \mathrm{K}(d, t-1), 1 \cdot \mathrm{K}(d$, $t-1), 2 \cdot \mathrm{K}(d, t-1), \ldots$, and $\left(b_{t-1}-1\right) \cdot \mathrm{K}(d, t-1)$, contained in the IK $(d, t)$. Clearly, $O\left(b_{t-1}\right)^{t-1}, 0^{t}, 1\left(b_{t-1}\right)^{t-1}$, and $1^{t}$ are all $(t-1)$ frontiers. We construct two node-disjoint paths according to the following two cases.

Case 1. $b_{t-1}=2$. 
By Lemma 4.1 , there is one hamiltonian path between $O\left(b_{t-1}\right)^{t-1}$ and $O^{t}$ in $0 \cdot \mathrm{K}(d, t-1)$. Likewise, there is one hamiltonian path hetween $1\left(b_{t-1}\right)^{t-1}$ and $1^{t}$ in $1 \cdot K(d, t-1)$. These two paths are node-disjoint, and they contain every node of the $\operatorname{IK}(d, t)$ exactly once.

Case 2. $b_{t-1}>2$.

Note that $0 \cdot \mathrm{K}(d, t-1)$ is composed of $00 \cdot \mathrm{K}(d, t-2)$, $01 \cdot \mathrm{K}(d, t-2), 02 \cdot \mathrm{K}(d, t-2), \ldots$, and $0(d-1) \cdot \mathrm{K}(d, t-2)$, and $1 \cdot \mathrm{K}(d, t-1)$ is composed of $10 \cdot \mathrm{K}(d, t-2), 11 \cdot \mathrm{K}(d, t-2)$, $12 \cdot \mathrm{K}(d, t-2), \ldots$, and $1(d-1) \cdot \mathrm{K}(d, t-2)$. A path between 0( $\left.b_{t-1}\right)^{t-1}$ and $0^{t}$ is constructed as follows:

$$
\begin{aligned}
& O\left(b_{t-1}\right)^{t-1} \rightarrow_{(\mathrm{H}, t-2)} O b_{t-1}\left(b_{t-1}+1\right)^{t-2} \rightarrow O\left(b_{t-1}+1\right)\left(b_{t-1}\right)^{t-2} \\
& \rightarrow(\mathrm{H}, t-2) \quad 0\left(b_{t-1}+1\right)\left(b_{t-1}+2\right)^{t-2} \rightarrow 0\left(b_{t-1}+2\right)\left(b_{t-1}+1\right)^{t-2} \\
& \rightarrow_{(\mathrm{H}, t-2)} \cdots \rightarrow_{(\mathrm{H}, t-2)} \mathrm{O}(d-1) 0^{t-2} \rightarrow 00(d-1)^{t-2} \rightarrow_{(\mathrm{H}, t-2)} \mathrm{O}^{t},
\end{aligned}
$$

where $\rightarrow$ indicates a flipping link and $\rightarrow_{(\mathrm{H}, t-2)}$ indicates a hamiltonian path in a $\mathrm{K}(d, t-2)$. The hamiltonicity is assured by Lemma 4.1. Actually this path contains every node of 0 $b_{t-1} \cdot \mathrm{K}(d, t-2), 0\left(b_{t-1}+1\right) \cdot \mathrm{K}(d, t-2), 0\left(b_{t-1}+2\right) \cdot \mathrm{K}(d, t-2), \ldots$, $0(d-1) \cdot \mathbf{K}(d, t-2)$, and $00 \cdot \mathbf{K}(d, t-2)$ exactly once.

On the other hand, a path between $1\left(b_{t-1}\right)^{t-1}$ and $1^{t}$ is constructed as the concatenation of the following four paths:

(1) $\mathrm{I}\left(b_{t-1}\right)^{t-1} \rightarrow(\mathrm{H}, t-2) 1 b_{t-1}\left(b_{t-1}+1\right)^{t-2} \rightarrow 1\left(b_{t-1}+1\right)\left(b_{t-1}\right)^{t-2}$

$\rightarrow_{(\mathrm{H}, t-2)} 1\left(b_{t-1}+1\right)\left(b_{t-1}+2\right)^{t-2} \rightarrow 1\left(b_{t-1}+2\right)\left(b_{t-1}+1\right)^{t-2}$

$\rightarrow(\mathrm{H}, t-2) \quad \cdots \quad \rightarrow(\mathrm{H}, t-2) \quad 1(d-1) 0^{t-2} \rightarrow 10(d-1)^{t-2}$

$\rightarrow(\mathrm{H}, t-2) \quad 10^{t-1} \rightarrow 01^{i-1}$;

(2) $01^{t-1} \rightarrow_{(\mathrm{HI}, t-2)} 012^{t-2} \rightarrow 021^{t-2} \rightarrow_{(\mathrm{H}, t-2)} 023^{t-2}$ $\rightarrow 032^{t-2} \rightarrow_{(\mathrm{H}, t-2)} \quad \cdots \rightarrow_{(\mathrm{H}, t-2)} \quad 0\left(b_{t-1}-2\right)\left(b_{t-1}-1\right)^{t-2}$ $\rightarrow \mathrm{O}\left(b_{t-1}-1\right)\left(b_{t-1}-2\right)^{t-2} \rightarrow(\mathrm{H}, t-2) \mathrm{O}\left(b_{t-1}-1\right)^{t-1} \rightarrow\left(b_{t-1}\right.$ 1) $0^{t-1}$

(3) $\left(b_{t-1}-1\right) 0^{t-1} \rightarrow_{(\mathrm{H}, t-1)}\left(b_{t-1}-1\right)\left(b_{t-1}-2\right)^{t-1} \rightarrow\left(b_{t-1}-2\right)\left(b_{t-1^{-}}\right.$ $1)^{t-1} \rightarrow_{(\mathrm{H}, t-1)}\left(b_{t-1}-2\right)\left(b_{t-1}-3\right)^{t-1} \rightarrow_{(\mathrm{H}, t-1)} \cdots \rightarrow_{(\mathrm{H}, t-1)}$ $32^{t-1} \rightarrow 23^{t-1} \rightarrow(\mathrm{H}, t-1) 21^{t-1} \rightarrow 12^{t-1}$;

(4) $12^{t-1} \rightarrow_{(\mathrm{H}, t-2)} 123^{t-2} \rightarrow 132^{t-2} \rightarrow_{(\mathrm{H}, t-2)} 134^{t-2} \rightarrow$ $143^{t-2} \rightarrow_{(\mathrm{H}, t-2)} \quad \cdots \quad \rightarrow_{(\mathrm{H}, t-2)} \quad 1\left(b_{t-1}-2\right)\left(b_{t-1}-1\right)^{t-2} \rightarrow$ $1\left(b_{t-1}-1\right)\left(b_{t-1}-2\right)^{t-2} \rightarrow_{(\mathrm{H}, t-2)} 1\left(b_{t-1}-1\right) t^{t-2} \rightarrow 11\left(b_{t-1^{-}}\right.$ $1)^{x-2} \rightarrow(\mathrm{H}, t-2) .1^{t}$

where the hamiltonicity is assured by Lemma 4.1. Path (1) contains all nodes of $1 b_{t-1} \cdot \mathrm{K}(d, t-2), 1\left(b_{t-1}+1\right) \cdot \mathrm{K}(d, t-2)$, $\ldots, 1(d-1) \cdot \mathrm{K}(d, t-2)$, and $10 \cdot \mathrm{K}(d, t-2)$. Path (2) contains all nodes of $01 \cdot \mathrm{K}(d, t-2), 02 \cdot \mathrm{K}(d, t-2), \ldots$, and $0\left(b_{t-1}-1\right) \cdot \mathrm{K}(d, t-$ 2). Path (3) contains all nodes of $\left(b_{t-1}-1\right) \cdot \mathrm{K}(d, t-1),\left(b_{t-1}-2\right.$ )$\cdot \mathrm{K}(d, t-1), \ldots$, and $2 \cdot \mathrm{K}(d, t-1)$. Path (4) contains all nodes of $12 \cdot \mathrm{K}(d, t-2), 13 \cdot \mathrm{K}(d, t-2), \ldots, 1\left(b_{t-1}-1\right) \cdot \mathrm{K}(d, t-2)$, and $11 \cdot \mathrm{K}(d, t-2)$. All nodes appear in these paths exactly once. It is not difficult to check that the two paths we have constructed between $O\left(b_{t-1}\right)^{t-1}$ and $O^{t}$ and between $1\left(b_{t-1}\right)^{t-1}$ and $1^{t}$ are node-disjoint, and they contain every node of the $\operatorname{IK}(d, t)$ exactly once. To illustrate the construction, Figure 4 shows two node-disjoint paths, one between 033 and 000 and the other between 133 and 111 , in $\operatorname{IK}(4,3)$ with coefficient vector $(3 . *)$.

Q.E.D.

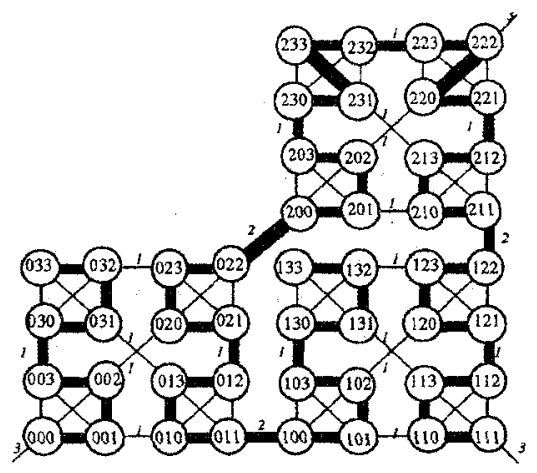

Figure 4. Two node-disjoint paths, one between 033 and 000 and the other between 133 and 111 in $\operatorname{IK}(4,3)$ with coefficient vector $(3, *)$.

A necessary condition for a hamiltinian graph is that its connectivity must be greater than 1. In the following, we show that the latter is also a sufficient condition for a hamiltonian $\operatorname{IK}(d, t)$.

Theorem 4.1. An $\operatorname{IK}(d, t)$, where $d \geq 3$, is hamiltonian if its connectivity is greater than 1 .

Proof. Suppose $\left(b_{t-1}, b_{t-2}, \ldots, b_{i}, *\right)$ is the coefficient vector of the IK $(d, t)$ and $R_{i}^{t-1}>1$. If $i=t-1$, by Theorem 3.1 we have $b_{t-1} \geq 3$. The $\operatorname{IK}(d, t)$ is composed of $b_{t-1} \mathrm{~K}(d, t-1)$ 's that are connected as a $b_{i-1}$-node complete graph. By the aid of Lemma 4.1, it is not difficult to see that there exists a hamiltonian cycle in the $\operatorname{IK}(d, t)$. So, in the rest of the proof, we assume $1 \leq i<t-1$. By Theorem 3.1 we have $b_{t-1} \geq 2$ and $b_{i} \geq 2$. By Lemma 4.2 , there exists a path between $0\left(b_{t-1}\right)^{t-1}$ and $1\left(b_{t-1}\right)^{t-1}$ which contains every node of $G_{t-1}$ exactly once, and there exists a path between $b_{t-1} b_{t-2 \ldots} \ldots b_{t+1} 0^{i+1}$ and $b_{t-1} b_{t-}$ $2 \ldots b_{i+1} 1^{i+1}$ which contains every node of $G_{i}$ exactly once. Since $R_{i}^{i-1}>1$, we have $b_{m} \geq 1$ for all $i<m<t-1$. A hamiltonian cycle in the $\operatorname{IK}(d, t)$ is constructed according to the following two cases.

Case 1. $b_{m} \geq 2$ for all $i<m<t-1$.

Lemma 4.3 assures that for $i<m<t-1$, there exist two node-disjoint paths in $G_{m}$, one between $U_{m, 0}=b_{t-1} b_{t-2} \ldots$ $b_{m+1} O\left(b_{m}\right)^{m}$ and $V_{m, 0}=b_{t-1} b_{t-2 \ldots} b_{m+1} 0^{m+1}$ and the other between $U_{m, 1}=b_{t-1} b_{t-2} \ldots b_{m+1} 1\left(b_{m}\right)^{m}$ and $V_{m, 1}=b_{t-1} b_{t-2} \ldots$ $b_{m+1} 1^{m+1}$, such that they contain every node of $G_{m}$ exactly once. A hamiltonian cycle in the $\operatorname{IK}(d, t)$ is thus formed as shown in Figure 5(a), where 
(a) $\left(0\left(b_{t-1}\right)^{t-1}, V_{t-2,0}\right)$ and $\left(1\left(b_{t-1}\right)^{t-1}, V_{t-2,1}\right)$ define two $(t-1)-$ flipping links;

(b) $\left(U_{i+1,0}, b_{t-1} b_{t-2} \ldots b_{i+1} 0^{i+1}\right)$ and $\left(U_{i+1,1}, b_{t-1} b_{t-2} \ldots\right.$ $\left.b_{i+1} l^{i+1}\right)$ define two $(i+1)$-tlipping links;

(C) $\left(U_{m, 0}, V_{m-1,0}\right)$ and $\left(U_{m, 1}, V_{m-1,1}\right)$ define two $m$-flipping links, where $i+i<m<t-1$.

Case 2. $b_{m}=1$ for one or more $m$ 's between $i$ and $t-1$.

We assume $b_{m}=1$ for exactly one $m$. The extension to multiple $m$ 's is very straightforward. According to Lemma 4.1 , there exists a path between $X=b_{t-1} b_{t-2} \ldots b_{m+1} 01^{m}$ and $Y=b_{t-1} b_{t-2} \ldots b_{m+1} 0^{m+1}$ which contains every node of $G_{m}$ exactly once. As shown in Figure $5(b)$, there is an $(m+1)$. nipping link between $U_{m+1,0}$ and $Y$, an $m$-flipping link between $X$ and $V_{m-1,0}$, and a jumping (m+1)-flipping link between $U_{m+1,1}$ and $V_{m-1,1}$. A hamiltonian cycle in the $\operatorname{IK}(d$, t) can be constructed similar to Case 1 .

Q.E.D.

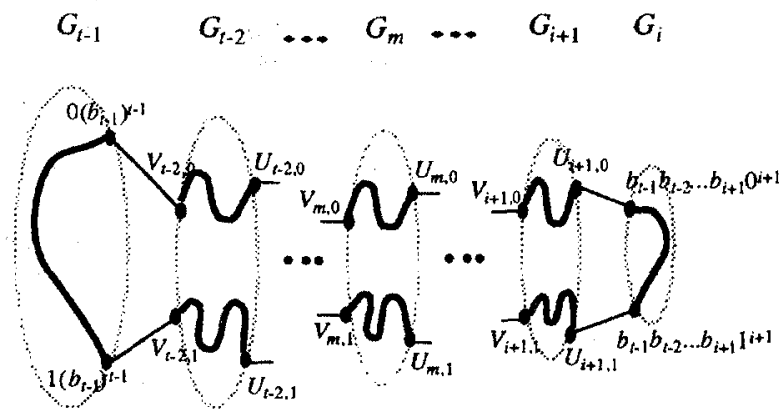

(a)

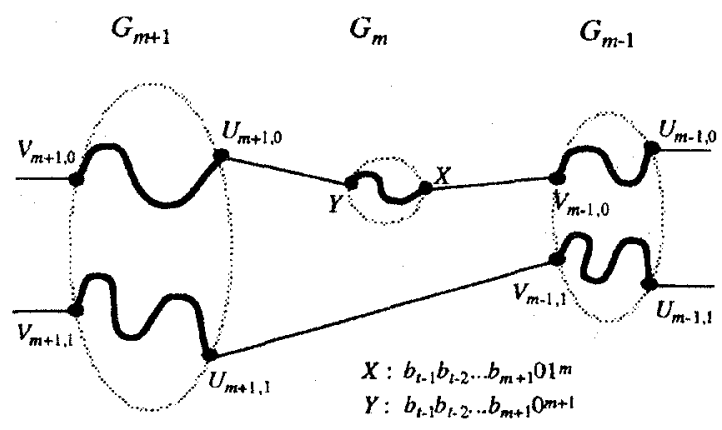

(b)

Figure 5. The proof of Theorem 4.1. (a) Case 1. (b) Case 2.

Theorem 4.1 guarantees a hamiltonian cycle in $\operatorname{IK}(d, t)$ with $d \geq 3$ if its connectivity is greater than 1 . For $\operatorname{IK}(d, t)$ with connectivity 1 , there is no hamiltonian cycle, and there is not necessarily a hamiltonian path. For example, no hamiltonian path exists in $\operatorname{IK}(4,4)$ with coefficient vector $(1,2,1, *)$. In what foliows, we identify the class of $\operatorname{IK}(d, t)$ s with connectivity 1 which contain a hamiltonian path.

For $\operatorname{IK}(d, t)$ with coefficient vector $\left(b_{t-1}, b_{t-2}, \ldots, b_{i,}, 1\right.$ and $R_{i}^{r-1}=1$, we can partition it into blocks. $G_{k}$ is a block if $b_{k} \neq 0$ and $\left(R_{k}^{k+1}=1\right.$ or $\left.b_{k+1}=0\right)$ and $\left(R_{k-1}^{k}=1\right.$ or $\left.b_{k-1}=0\right)$. $G_{m}+G_{m-1}+\ldots+G_{n}$, where $b_{m} \neq 0, b_{n} \neq 0$ and $m>n$, is a block if $R_{n}^{m}>1$ and $\left(R_{n}^{m+1}=1\right.$ or $\left.b_{m+1}=0\right)$ and $\left(R_{n+1}^{m}=1\right.$ or $\left.b_{n+1}=0\right)$. The partition can be easily done by examining the coefficient vector. As an illustrative example let us consider $\operatorname{IK}(6,10)$ with coefficient vector $(1,2,1,2,0,1, *)$. By Lemma 3.4, there are two jumping flipping links. One is between the 2nd $\mathrm{K}(6,8)$ within $G_{8}$ and the 2nd $\mathrm{K}(6,6)$ within $G_{6}$, and the other is between the first $\mathrm{K}(6,6)$ within $G_{6}$ and the first $\mathrm{K}(6$, 4) within $G_{4}$. Clearly $G_{9}$ and $G_{4}$ are two blocks because $R_{8}^{9}=1$ and $b_{5}=0$, respectively. $G_{8}+G_{7}+G_{6}$ is another block because $R_{6}^{8}=2, R_{6}^{9}=1$, and $b_{5}=0$. Hence $\operatorname{IK}(6,10)$ with coefficient vector $(1,2,1,2,0,1, *)$ can be partitioned into $\left\{G_{9}, G_{8}+G_{7}+G_{6}, G_{4}\right\}$. Intuitively, if each $G_{j}(4 \leq j \leq 9)$ with $b_{f} \neq 0$ is regarded as a node, then the flipping links between $G_{9}$ and $G_{8}$ and between $G_{6}$ and $G_{4}$ are two bridges [2], and each block is either a single node or a maximal biconnected component in the resulting graph. The following two lemmas have proven in [20].

Lemma 4.4.[20] An $\operatorname{IK}(d, t)$ with connectivity 1 contains a hamiltonian path if it consists of one or two blocks.

Lemma 4.5.[20] Consider an $\operatorname{IK}(d, t)$ with coefficient vector $\left(b_{t-1}, b_{t-2}, \ldots, b_{i}, *\right)$ and $R_{i}^{t-1}=1$ that contains three or more blocks. There is a hamiltonian path in the $\operatorname{IK}(d, t)$ if and only if for each block, say $G_{m}+G_{m-1}+\ldots+G_{n}$, in the $\operatorname{IK}(d$, $t)$, no $b_{r+1}, b_{r}, b_{r-1}, \ldots, b_{s}, b_{s-1}$ exist such that $b_{r+1} \in\{0,1\}$, $b_{r}=b_{r-1}=\ldots=b_{s}=2, b_{s-1} \in\{0,1\}$, and $r-s+1$ is odd, where $m \neq i-1, n \neq i$, and $n \leq s \leq r \leq m$.

Combining Lemmas 4.4 and 4.5 , we have a necessary and sufficient condition for a hamiltonian path in an $\operatorname{IK}(d, t)$ with connectivity 1 .

Theorem 4.2. For $\operatorname{IK}(d, t)$ with coefficient vector $\left(b_{t-1}\right.$, $\left.b_{t-2}, \ldots, b_{i}, *\right)$ and $R_{i}^{t-1}=1$, it contains a hamiltonian path if and only if either of the following two conditions holds:

(1) it contains one or two blocks;

(2) for each block, say $G_{m}+G_{m-1}+\ldots+G_{n}$, in the $\operatorname{IK}(d, t)$, no $b_{r+1}, b_{r}, b_{r-1}, \ldots, b_{s}, b_{s-1}$ exist such that $b_{r+1} \in\{0$. 1\}, $b_{r}=b_{r-1}=\ldots=b_{s}=2, b_{s-1} \in\{0,1\}$, and $r-s+1$ is odd, where $m \neq t-1, n \neq i$, and $n \leq s \leq r \leq m$.

\section{Concluding Remarks}

Deriving topological properties for incomplete networks is far more difficult than for complete networks. The reason is 
that complete networks of different sizes preserve great topological similarity, whereas incomplete networks may have a significant difference in their topologies. For example, $\mathrm{K}(\boldsymbol{d}$, t) looks very similar to $\mathrm{K}(d, t-1)$, whereas two $\operatorname{IK}(d, t)$ 's with different coefficient vectors may look very unlike in their topologies. Many of topological properties of the incomplete star networks [13], [16] are still unknown, although they have been well solved for the star networks [1]. Most of the results obtained for the incomplete star networks are restricted to a special case: $N=c \cdot k$ !, where $N$ is the number of nodes.

In this paper, we have shown it very convenient to represent the structure of an incomplete WK-recursive networks by a "multistage-like" graph $G_{t-1}+G_{t-2}+\ldots+G_{i}$. This representation provides a uniform look at the incomplete WK-recursive networks, and thus facilitates the derivation of many properties. By the aid of this representation, we have computed the connectivities and hamiltonicity. Moreover, we have suggested a tight upper bound on the diameters. The methods adopted in this paper are different from Chen and Duh's for the WK-recursive networks [4]. Readers who are interested in the incomplete WK-recursive networks are refered to [20] and [21] for more results. Precisely, using the prune-and-search technique a linear-time algorithm for computing the diameters can be found in [20], and a distributed shortest-path routing algorithm can be found in [21].

\section{References}

[1] S. B. Akers and B. Krishnamurthy, "A group-theoretic model for symmetry interconnection networks," IEEE Trans. on Computers, vol. 38, no. 4, pp. 555-566, 1989.

[2] J. Bondy and U. Murthy, Graph Theory with Applications, Macmillan Press, 1976.

[3] F. Buckley and F. Harary, Distance in Graph, AddisonWesley, 1990.

[4] G. H. Chen and D. R. Duh, "Topological properties, communication, and computation on WK-recursive networks," Networks, vol. 24, no. 6, pp. 303-317, 1994.

[5] P. Corbett, "Rotator graphs: an efficient topology for point-to-point multiprocessor networks," IEEE Trans. on Parallel and Distributed Systems, vol. 3, no. 5, pp. 622-626, 1992.

[6] D. R. Duh and G. H. Chen, "Topological properties of WK-recursive networks," $J$. of Parallel and Distributed Computing, vol. 23, pp. 468-474, 1994.

[7] R. Fernandes, "Recursive interconnection networks for multicomputer networks," in Proc. of the Int. Conf.on Parallel Processing, vol. 1, pp. 76-79, 1992.

[8] R. Fernandes and A. Kanevsky, "Hierarchical WKrecursive topologies for multicomputer systems," in Proc. of the Int. Conf.on Parallel Processing, vol. 1 , pp. 315-318, 1993.
[9] R. Fernandes and A. Kanevsky, "Substructure allocation in recursive interconnection networks," in Proc. of the Int. Conf.on Parallel Processing. vol. 1. 1993, pp. 319-322.

[10] R. Fernandes, D. K. Griesen, and A. Kanevsky. "Efficient routing and broadcasting in recursive interconnection networks," in Proc. of the Int. Conf.on Parallel Processing, 1994, pp. 51-58.

[11] R. Fernandes, D. K. Friesen, and A. Kanevsky, "Embedding rings in recursive networks," in Proc. of Int. Symp. on Parallel and Distributed Processing. Oct. 1994, pp. 273-280.

[12] H. P. Katseff, "Incomplete hypercubes," IEEE Trans. on Computers, vol. C-37, no. 5, pp. 604-608, 1988.

[13] S. Latifi and N. Bagherzadeh, "Incomplete star: an incrementally scalable network based on the star graph," IEEE Trans. on Parallel and Distributed Systems, voi. 5, no. 1, pp. 97-102, 1994.

[14] S. Ponnuswamy and V. Chaudhary, "Embedding of cycles in rotator and incomplete rotator graphs," in Proc. of Int. Symp. on Parallel and Distributed Processing, Oct. 1994, pp. 603-610.

[15] F. P. Preparata and J. Vuillemin, "The cube-connected cycles: a versatile network for parallel computation," Communications of the ACM, vol. 24, no. 5, pp. 300$309,1981$.

[16] C. P. Ravikumar, A. Kuchlous, and G. Manimaran, "Incomplete star graph: an economical fault-tolerant interconnection network," in Proc. of Int..Conf. on Parallel Processing, vol. 1, 1993, pp. 83-90.

[17] Y. Saad and M. H. Schultz, "Topological properties of hypercubes," IEEE Transactions on Computers, vol. 37, no. 7, pp. 867-872, 1988.

[18] M. R. Samatham and D. K. Pradhan, "The de Bruijn multiprocessor networks: a versatile parallel processing and sorting networks for VLSI," IEEE Trans. on Computers, vol. 38, pp. 567-581, 1989.

[19] H. S. Stone, "Parallel processing with the perfect shuffle," IEEE Trans. on Computers, vol. 20, no. 2, pp. 153.161, 1971

[20] M. Y. Su, G. H. Chen, and D. R. Duh, "Topological properties of incomplete WK-recursive networks," Tech. Rep. NTUCSIE 95-06, National. Taiwan University, Taipei, Taiwan, March 1995.

[21] M. Y. Su, G. H. Chen, and D. R. Duh, "A shortestpath routing algorithm for the incomplete WK-recursive networks," Tech. Rep. NTUCSIE 95-07, National Taiwan University, Taipei, Taiwan, July 1995.

[22] G. D. Vecchia and C. Sanges, "A recursively scalable network VLSI implementation," Future Generation Computer Systems, vol. 4, no. 3, pp. 235-243, 1988.

[23] G. D. Vecchia and C. Sanges, "An optimized broadcasting technique for WK-recursive topologies," Future Generation Computer Systems, vol. 4, no. 3, pp. 353-357, $1989 / 90$. 